\title{
RECOMENDACIONES CONDICIONADAS DE FERTILIZACIÓN CON NITRÓGENO Y FÓSFORO PARA MAÍZZ USANDO UN MODELO CUADRÁTICO EN LA PROVINCIA DE CHIRIQUÍ, PANAMÁ*
}

\author{
Gustavo Sain**, Miguel Angel Acosta***
}

\begin{abstract}
RESUMEN
Mediante un modelo general de respuesta cuadrático se generan recomendaciones sobre dosis óptimas económicas de nitrógeno $(\mathrm{N})$ y fósforo $(\mathrm{P})$ en maíz en dos dominios de recomendación en la Provincia de Chiriquí, Panamá. Para el dominio de recomendación 1 (DR1), caracterizado por alto contenido inicial de $\mathrm{P}$ en el suelo y bajos niveles de materia orgánica (MO), las dosis óptimas fueron de $184 \mathrm{~kg} \mathrm{~N} / \mathrm{ha} \mathrm{y} \mathrm{la} \mathrm{no}$ aplicación de P. Para el dominio de recomendación 2 (DR2), caraterizado por bajo contenido inicial de $\mathrm{P}$ y altos niveles de

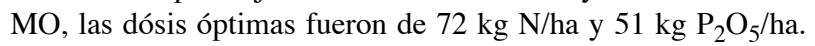
La rentabilidad de la inversión en fertilizantes en el DRl fuesignificativamente superior que aquella alcanzada en el DR2. Para que la fertilización fuera rentable, la tasa m ínima de retorno aceptable debe ser como máximo de $42 \%$ para los agricultores en el DR1 y de $93 \%$ para aquellos pertenecientes al DR2.
\end{abstract}

\begin{abstract}
A general quadratic response model is used to find optimal rates of nitrogen $(\mathrm{N})$ and phosphorous $(\mathrm{P})$ for maize in two recommendation domains in the Chiriqui Province, Panamá. For the recommendation domain 1 (RD1), characterized by high initial content for $\mathrm{P}$ in the soil and a low level of organic matter (OM), optimal economic rates were 184 $\mathrm{kg} \mathrm{N} / \mathrm{ha}$ and no $\mathrm{P}$ application. For the RD2, low $\mathrm{P}$ and high level of $\mathrm{MO}$, the optimal rates were $72 \mathrm{~kg} \mathrm{~N} / \mathrm{ha}$ and $51 \mathrm{~kg} \mathrm{P}_{2} \mathrm{O}_{5} / \mathrm{ha}$.

For the DRl the profitability of fertilizer application was found to be significantly superior to that found in RD2. For fertilization to be profitable in the RD1 the minimum rate of return acceptable to farmers must be inferior to $42 \%$, while in RD2 this rate must be inferior to $93 \%$.
\end{abstract}

\section{INTRODUCCCIÓN}

En la mayor ía de los programas de investigación en fincas (IFA) la formulación de recomendaciones se realiza mediante la evaluación económica de los resultados experimentales usando el método del análisis discreto sobre la información generada en un dominio de recomendación (para un tratamiento detallado del análisis discreto véase CIMMYT, 1988).

Al mismo tiempo el análisis económico continuo a través de la estimación de una función general de respuesta presenta un conjunto de ventajas tales como la mejor interpretación de los datos y mayor capacidad y facilidad para analizar un conjunto de datos provenientes de distintos sitios y/o años.
Una función de respuesta es una relación cuantitativa que describe el rendimiento máximo obtenible a partir de diferentes combinaciones de nutrimentos y otros factores de producción. La función de respuesta se representa como:

[1] $Y_{t}=f\left(N_{i t}, X_{j t}, S_{k t}, R_{l t}\right)$

Donde $Y_{t}$ representa el rendimiento en el período t, $\mathrm{N}_{\mathrm{it}}(\mathrm{i}=1, \ldots \mathrm{I})$ es un vector de I nutrimentos disponibles para la planta en el período $t ; X_{j t}(j=1, \ldots J)$ es un vector de insumos y servicios manejados a discreción del agricultor, tal como es variedad, y control de malezas. $\mathrm{S}_{\mathrm{kt}}$ $(\mathrm{k}=1, \ldots \mathrm{K})$ representa un vector de $\mathrm{K}$ variables que pueden conocerse o no al comienzo del período

\footnotetext{
* Trabajo presentado en la XXXVIII reunión anual del PCCMCA, Managua, Nicaragua 23-27 Marzo de 1992. Los autores desean agradecer al Ing. Mario Jauregui y Hector Barreto por sus comentarios y sugerencias hechas al trabajo.

** Economista Regional del Programa Regional para Centro América y el Caribe del CIMMYT.

*** Especialista en Agronomía, Sub Centro de Santa Fé del IDIAP, Panamá.
} 
productivo t, pero que el agricultor no puede modificar, por ejemplo, el tipo y calidad del suelo. Finalmente, $\mathrm{R}_{\mathrm{lt}}$ $(1=1, \ldots \mathrm{L})$ es un vector de $\mathrm{L}$ variables climáticas fuera del control del agricultor que ocurren durante el ciclo productivo $\mathrm{t}$ y afectan la respuesta del cultivo. La cantidad y distribución de la precipitación es un buen ejemplo de este último tipo de variables.

Un dominio de recomendación se detine como un grupo de agricultores cuyas circunstancias son lo suficientemente similares como para que una misma recomendación sea apropiada para todos ellos. La estrategia de la investigación en fincas usa este concepto para obtener recomendaciones apropiadas a las circunstancias de los agricultores objetivos dentro de cada dominio de recomendación. Las variables que se usan normalmente para el establecimiento de dominios pertenecen a las clases comprendidas por $\mathrm{X}_{\mathrm{jt}}$ y $\mathrm{S}_{\mathrm{kt}}$ en la ecuación [1]. En términos del análisis de la respuesta a la fertilización, un modelo general busca incorporar componentes de los vectores $\mathrm{X}, \mathrm{S}$ y $\mathrm{R}$ de manera que a través de la estimación de una sola función $\mathrm{Y}=$ $\mathrm{f}\left(\mathrm{N}_{\mathrm{i}}, \mathrm{X}_{\mathrm{j}}, \mathrm{S}_{\mathrm{k}}, \mathrm{R}_{\mathrm{l}}\right)$ se obtengan recomendaciones sobre las dosis óptimas de nutrimentos $\mathrm{N}_{\mathrm{i}}$ condicionadas a los valores de estas variables de sitio.

El objetivo de este trabajo es presentar un modelo general para la formulación de recomendaciones de fertilización con nitrógeno $(\mathrm{N})$ y fósforo $(\mathrm{P})$, condicionadas por variables de sitio usando la forma funcional cuadrática. El método se aplica a los resultados de tres experimentos de fertilización con $\mathrm{N}$ y $\mathrm{P}$ en distintos suelos del suroeste de la Provincia de Chiriquí en Panamá (Acosta et al. 1991).

El método permite una mejor interpretación de la información obtenida en los experimentos comparada con la obtención de recomendaciones de dosis óptimas de P y $\mathrm{N}$ basadas en la informacion de cada localidad o basadas en el análisis discreto de presupuesto parcial.

\section{MATERIALES Y MÉTODOS}

El área de trabajo se ubica en la llanura costera del Pacífico de la Provincia de Chiriquí abarcando desde el distrito de Barú hasta David, Panamá. Esta llanura posee un área cultivada de más de 10000 ha. Aunque tradicionalmente esta ha sido un área arrocera, en los últimos 10 años se ha producido un reemplazo de la superficie dedicada al arroz por maíz. Actualmente los sistemas predominante son:

1- Arroz en primera coa - maíz en segunda coa y

2- Maíz en primera coa - frijol de bejuco en segunda coa.

Se estima que en 1989 el área cultivada con maíz fue de aproximadamante 3200 ha, con rendimientos que en promedio no superaron los $2300 \mathrm{~kg}$ por hectárea (Acosta et al. 1991).

Aunque los productores del área están familiarizados con el uso de fertilizantes, las dosis que aplican se basan en su experiencia y la de los extensionistas con el cultivo del arroz. De acuerdo a los resultados del diagnóstico realizado en el área, los agricultores realizan una fertilización con dosis aproximadas de $80 \mathrm{~kg} \mathrm{~N} / \mathrm{ha}, 45 \mathrm{~kg}$ $\mathrm{P} / \mathrm{ha}$ y $20 \mathrm{~kg} \mathrm{~K} / \mathrm{ha}$.

Con el objetivo de obtener información sobre la respuesta del cultivo a la aplicación de $\mathrm{N}$ y $\mathrm{P}$ y sobre las dosis óptimas económicas a ser recomendadas a los agricultores del área; en la primera coa de 1989 se llevó a cabo un experimento en tres localidades del área: Corozal, Guarumal, y Los Angeles de Sioguí. Estas localidades se encuentran ubicadas en los distritos de David, Alanje, y Bugaba respectivamente.

Los ensayos se sembraron en campos de agricultores colaboradores. La siembra se realizó a chuzo, entre la última semana de junio y la primera de julio de 1989, utilizando la variedad Alanje 1.

Las dosis experimentales de $\mathrm{N}$ fueron $0,50,100,150$ y $200 \mathrm{~kg} \mathrm{~N} / \mathrm{ha}$ combinadas con cinco dosis de $\mathrm{P}_{2} \mathrm{O}_{5}: 0,80$, 160, 240, Y $340 \mathrm{~kg} \mathrm{P}_{2} \mathrm{O}_{5} /$ ha. Todos los tratamientos se realizaron en dos aplicaciones y se establecieron en bloques completos al azar con cuatro repeticiones arreglados en un factorial incompleto $5^{2}$ (Figura 1).

La unidad experimental consistió en cuatro surcos de $5 \mathrm{~m}$ de largo separados a $0.80 \mathrm{~m}$ entre si y $0,20 \mathrm{~m}$ entre plantas lo que dió una población teórica de 62500 pl/ha.

Una variable importante condicionando la respuesta a la fertilización es el contenido de nutrimentos disponibles en el suelo. El Cuadro 1 resume las 
principales características de los suelos en donde se realizaron los ensayos.

El modelo general propuesto en este trabajo es un modelo cuadrático que incorpora una variable de sitio D que toma los valor 1 para Guarumal (alto contenido de $\mathrm{P}$ inicial y bajo contenido de materia orgánica) y 0 para las otras dos localidades (bajo contenido de $\mathrm{P}$ inicial y alto contenido de materia orgánica). De esta manera se distinguen dos dominios de recomendación y se utilizan eficientemente los grados de libertad provistos por las observaciones agregadas.
1992). Los resultados alcanzados por el modelo seleccionado mediante esta prueba se comparan con aquellos que se hubieran obtenido si el modelo se hubiera ajustado a cada una de las

localidades y con los obtenidos por el análisis marginal discreto. Se realiza un análisis de la sensibilidad de las dosis óptimas obtenidas y de los beneficios netos por hectárea ante cambios en las relaciones de precios. Finalmente se examina la rentabilidad de la inversión en fertilizantes en cada uno de los dominios de recomendación identificados mediante el cálculo de las

Cuadro 1. Principales características químicas de los suelos donde se establecieron los experimentos. Chiriquí, Panamá, 1989.

\begin{tabular}{|c|c|c|c|c|c|c|c|c|}
\hline & \multirow{2}{*}{$\mathrm{pH}$} & $\mathrm{MO}$ & $\mathrm{P}$ & $\mathrm{K}$ & $\mathrm{Ca}$ & $\mathrm{Mg}$ & \multicolumn{4}{|c|}{$\mathrm{Al}$} \\
\cline { 3 - 8 } Localidades & & $\%$ & \multicolumn{4}{|c|}{$\mathrm{mg} / \mathrm{ml}$} \\
\hline Corozal & 6,2 & 11,52 & 3,55 & 172,3 & 5,04 & 4,07 & 0,04 \\
Guarumal & 6,2 & 5,53 & 20,9 & 194,4 & 4,07 & 2,72 & 0,04 \\
Los Angeles de Sioguí & 5,6 & 11,26 & 4,26 & 219,0 & 2,09 & 1,16 & 0,04 \\
\hline
\end{tabular}

Fuente: (1)

Se estiman cuatro modelos que permiten diferentes formas en que la variable de sitio modifica la respuesta a la fertilización.

Modelo básico:

$[2] \mathrm{y}=\mathrm{b}_{0}+\mathrm{b}_{1} \mathrm{~N}+\mathrm{b}_{2} \mathrm{P}+\mathrm{b}_{3} \mathrm{~N}^{2}+\mathrm{b}_{4} \mathrm{P}^{2}+\mathrm{b}_{5} \mathrm{NP}$

Modelo corto:

[3] $\mathrm{Y}=\mathrm{b}_{0}+\mathrm{b}_{1} \mathrm{~N}+\mathrm{b}_{2} \mathrm{P}+\mathrm{b}_{3} \mathrm{~N}^{2}+\mathrm{b}_{4} \mathrm{P}^{2}+\mathrm{b}_{5} \mathrm{NP}+\mathrm{b}_{6} \mathrm{D}$

Modelo intermedio:

[4] $\mathrm{Y}=\mathrm{b}_{0}+\mathrm{b}_{1} \mathrm{~N}+\mathrm{b}_{2} \mathrm{P}+\mathrm{b}_{3} \mathrm{~N}^{2}+\mathrm{b}_{4} \mathrm{P}^{2}+\mathrm{b}_{5} \mathrm{NP}$ $+\mathrm{b} 6 \mathrm{D}+\mathrm{b} 7 \mathrm{DN}+\mathrm{bsDP}$

Modelo completo:

$$
\begin{aligned}
{[5] \mathrm{y}=} & \mathrm{b}_{0}+\mathrm{b}_{1} \mathrm{~N}+\mathrm{b}_{2} \mathrm{P}+\mathrm{b}_{3} \mathrm{~N}^{2}+\mathrm{b}_{4} \mathrm{P}^{2}+\mathrm{b}_{5} \mathrm{NP} \\
& +\mathrm{b}_{6} \mathrm{D}+\mathrm{b}_{7} \mathrm{DN}+\mathrm{b}_{8} \mathrm{DP}+\mathrm{b}_{9} \mathrm{DN}^{2}+\mathrm{b}_{10} \mathrm{DP}^{2}+ \\
& \mathrm{b}_{11} \mathrm{DNP}
\end{aligned}
$$

En el modelo corto la variable de sitio D solo modifica el intercepto, en el intermedio modifica también los efectos lineales y en el completo se permite además la interacción con los efectos cuadráticos de $\mathrm{N}$ y $\mathrm{P}$ incluyendo la in ter acción de $\mathrm{N}$ y $\mathrm{P}$.

Los modelos se evalúan entre si mediante la prueba de F para comparación de modelos anidados (Gujarati funciones de beneficio neto $(\mathrm{BN})$ y de rentabilidad (TMR). Estas funciones expresan los beneficios netos y la tasa marginal de retorno respectivamente para niveles alternativos de inversión en fertilizantes a lo largo del sendero de expansión.

\section{RESULTADOS Y DISCUSIÓN}

El Cuadro 2 muestra los resultados obtenidos de la estimación de los parámetros de los cuatro modelos mediante el método de los cuadrados mínimos ordinarios (CMO).

Note que el ajuste a los datos agregados sin introducir ninguna variable adicional (modelo básico) explica solamente un $30 \%$ de la variabilidad total, debido a la respuesta diferencial en cada una de las localidades. Es decir que, aunque este modelo tiene 33 grados de libertad resultantes de 39 observaciones y 6 parámetros su poder explicativo es bajo.

La introducción de la variable de sitio D eleva significativamente el poder explicativo del modelo cuadrático ajustado. La prueba de selección de modelos anidados que se da inmediatamente después de la tabla 2 , 
indica que no existe ganancia adicional entre el modelo completo y el intermedio. Por lo que se adopta éste último para representar la respuesta del rendimiento de maíz a la fertilización con $\mathrm{N}$ en cada uno de los dos dominios de recomendación.

Cuadro 2. Parámetros estimados de los cuatro modelos propuestos.

\begin{tabular}{|c|c|c|c|c|}
\hline \multirow[t]{2}{*}{ Variables } & \multicolumn{4}{|c|}{ Modelos } \\
\hline & Básico & Corto & Intermedio & Completo \\
\hline Constante & 3067 & 3949 & 3467 & 3238 \\
\hline $\mathrm{N}$ & $\begin{array}{l}16,06 \\
(1,83)\end{array}$ & $\begin{array}{l}16,06 \\
(2,34) . .\end{array}$ & $\begin{array}{l}24,34 \\
(4,1) \ldots *\end{array}$ & $\begin{array}{l}37,48 \\
(3,91) \ldots\end{array}$ \\
\hline$P$ & $\begin{array}{c}5,96 \\
(1,09)\end{array}$ & $\begin{array}{c}5,96 \\
(1,39)\end{array}$ & $\begin{array}{c}3,80 \\
(1,02)\end{array}$ & $\begin{array}{c}1,07 \\
(0,18)\end{array}$ \\
\hline $\mathrm{N}^{2}$ & $\begin{array}{r}-0,055 \\
(-1,39)\end{array}$ & $\begin{array}{c}-0,055 \\
(-1,78)^{*}\end{array}$ & $\begin{array}{c}-0,055 \\
(-2,19)^{* *}\end{array}$ & $\begin{array}{c}-0,118 \\
(-2,73)^{*}\end{array}$ \\
\hline$p^{2}$ & $\begin{array}{l}-0,014 \\
(-0,93)\end{array}$ & $\begin{array}{l}-0,014 \\
(-1,2)\end{array}$ & $\begin{array}{r}-0,014 \\
(-1,48)\end{array}$ & $\begin{array}{l}-0,005 \\
(-0,29)\end{array}$ \\
\hline NP & $\begin{array}{c}0,01 \\
(0,52)\end{array}$ & $\begin{array}{r}0,001 \\
(0,66)\end{array}$ & $\begin{array}{r}0,001 \\
(0,82)\end{array}$ & $\begin{array}{r}0,007 \\
(0,32)\end{array}$ \\
\hline D & & $\begin{array}{c}-1323 \\
(-4,7)^{x \ldots}\end{array}$ & $\begin{array}{c}-600 \\
(-1,21)\end{array}$ & $\begin{array}{c}-256 \\
(-0,36)\end{array}$ \\
\hline DN & & & $\begin{array}{l}-12,42 \\
(-3,99)^{n *}\end{array}$ & $\begin{array}{l}-32,13 \\
(-2,74)\end{array}$ \\
\hline DP & & & $\begin{array}{c}3,24 \\
(1,69)^{*}\end{array}$ & $\begin{array}{c}7,35 \\
(1,00)\end{array}$ \\
\hline$D N^{2}$ & & & & $\begin{array}{c}0,095 \\
(1,79)^{*}\end{array}$ \\
\hline$D P^{2}$ & & & & $\begin{array}{r}-0,014 \\
(-0,69)\end{array}$ \\
\hline DNP & & & & $\begin{array}{r}0,005 \\
(0,19)\end{array}$ \\
\hline $\mathrm{R}^{2}$ & 0,30 & 0,59 & 0,75 & 0,77 \\
\hline g.l. & 33 & 32 & 30 & 27 \\
\hline
\end{tabular}

Los resultados del test de selección de modelos anidados propuestos por Gujarati (1992), citados por Jauregui y Sain 1991, fueron:

\section{Corto versus básico}

$$
\begin{gathered}
F=\frac{R_{c}{ }^{2}-R_{b}{ }^{2} / k_{c}-k_{b}}{\left(l-R_{c}{ }^{2}\right) /\left(n-k_{c}\right)} \\
F=\frac{(0,59-0,30) / 7-6}{0,41 / 32}=22,63 * * *
\end{gathered}
$$

\section{Intermedio versus corto}

$$
\begin{aligned}
& F=\frac{R_{i}{ }^{2}-R_{s}{ }^{2} / k_{i}-k_{s}}{\left(1-R_{i}{ }^{2}\right)-\left(n-k_{i}\right)} \\
& F=\frac{(0,75-0,59) / 9-7}{0,25 / 30}=9,6 * * *
\end{aligned}
$$

\section{Intermedio versus completo}

$$
\begin{gathered}
F=\frac{R_{c}{ }^{2}-R_{i}{ }^{2} / k_{c}-k_{i}}{\left(l-R_{c}{ }^{2}\right) /\left(n-k_{c}\right)} \\
F=\frac{(0,77-0,75) / 12-9}{0,41 / 32}=0,8116^{* *}
\end{gathered}
$$

donde, $\mathrm{R}_{\mathrm{b}}^{2}, \mathrm{R}_{\mathrm{c}}{ }^{2}, \mathrm{R}_{\mathrm{i}}^{2}$ y $\mathrm{R}_{\mathrm{co}}{ }^{2}$ representan el coeficiente de determinación de los modelos básico, corto, intermedio y completo respectivamente. Así mismo, $\mathrm{k}_{\mathrm{b}}$, $\mathrm{k}_{\mathrm{c}}, \mathrm{k}_{\mathrm{i}}, \mathrm{y} \mathrm{k}_{\mathrm{co}}$ corresponden al números de parámetros estimados en cada uno de estos modelos. Finalmente, $n$ representa el número de observaciones en la muestra.

El valor estimado de $\mathrm{F}$ se compara con el valor tabulado para los grados de libertad correspondientes. La hipótesis nula de que los modelos tienen el mismo valor explicativo se rechaza si el valor estimado de $\mathrm{F}$ es mayor que el tabulado. En este caso, la hipótesis nula no puede ser rechazada para la comparación entre el modelo intermedio y completo, por lo que se toma este como el más adecuado para representar la respuesta del cultivo a la fertilización.

De acuerdo al modelo intermedio estimado, las funciones de respuesta para cada uno de los valores de la variable son las siguientes:

Dominio Recomendación 1 (DR1). Bajo fósforo inicial, alta materia orgánica.

\section{$[6] \mathrm{Y}=2867+11.92 \mathrm{~N}+7,04 \mathrm{P}-0.055 \mathrm{~N}^{2}-0.014 \mathrm{P}^{2}+$ $0.001 \mathrm{NP}$}

Dominio Recomendación 2 (DR2). Alto fósforo inicial, baja materia orgánica.

$\begin{aligned} {[7] \mathrm{Y}=} & 3467+24.34 \mathrm{~N}+3.80 \mathrm{P}-0.055 \mathrm{~N}^{2}-0.014 \mathrm{P}^{2}+ \\ & 0.01 \mathrm{NP}\end{aligned}$

Los óptimos económicos para $\mathrm{N}\left(\mathrm{N}^{*}\right)$ y para $\mathrm{P}\left(\mathrm{P}^{*}\right)$ se calculan de la siguiente manera:

$$
\begin{gathered}
\text { [8] } N^{*}=\frac{2 b_{22}\left(r_{n}-b_{1}\right)-b_{12}\left(r_{p}-b_{2}\right)}{\left(4 b_{11} b_{22}-b_{12}^{2}\right)} \\
P^{*}=\frac{r_{p}-b_{2}-b_{12} N^{*}}{2 b_{22}}
\end{gathered}
$$


donde $r_{n}$ y $r_{p}$ representan las relaciones de precios para $\mathrm{N}$ y $\mathrm{P}$ respectivamente y se calculan de acuerdo a las fórmulas siguientes (3) y (6).

$$
\text { [9] } \begin{aligned}
r_{n} & =\frac{\left(P_{n}+C A\right)(l+R)}{P_{o}(l-a)} \\
r_{p} & =\frac{\left(P_{p}+C A\right)(1+R)}{\operatorname{Po}(1-\mathrm{a})}
\end{aligned}
$$

donde a es un coeficiente de ajuste de rendimientos, $\mathrm{P}_{\mathrm{n}}, \mathrm{P}_{\mathrm{p}}$ y $\mathrm{P}_{\mathrm{o}}$ son los precios de campo del nitrógeno, fósforo y maíz respectivamente; $\mathrm{CA}$ es el costo de aplicación del fertilizante; y $\mathrm{R}$ es la tasa mínima de retorno aceptable a los agricultores en el área.

Para su cálculo se usaron los valores siguientes: $\mathrm{a}=20 \% ; \mathrm{P}_{\mathrm{n}}=\$ 0.55 / \mathrm{kg} ; \mathrm{P}_{\mathrm{p}}=\$ 0.83 / \mathrm{kg} ; \mathrm{CA}=0.04 / \mathrm{kg}$. $\mathrm{P}_{\mathrm{o}}=\$ 0.225 \mathrm{jkg} ; \mathrm{R}=25 \%$ fueron:

Usando esta información los valores de $r_{n}$ y $r_{p}$

$\mathrm{r}_{\mathrm{n}}=\frac{(0.55+0.04)(1+0.25)}{0,225(1-0,20)}=4,1 \quad \mathrm{y}$

$\mathrm{r}_{\mathrm{p}}=\frac{(0.83+0.04)(1+0.25)}{0,225(1-0,20)}=6,04$

Es decir que se necesitarían 4 y $6 \mathrm{~kg}$ de maíz para comprar $1 \mathrm{~kg}$ de $\mathrm{N} \mathrm{y}_{2} \mathrm{O}_{5}$, respectivamente.

El Cuadro 3 presenta las dosis económicas obtenidas a través del intermedio para las tres localidades.

Cuadro 3. Dosis óptimas económicas obtenidas de

\begin{tabular}{|c|c|c|c|c|c|c|}
\hline \multirow{3}{*}{$\begin{array}{l}\text { Dominios de } \\
\text { recomendación }\end{array}$} & \multicolumn{6}{|c|}{ Dosis óptimas $(\mathrm{kg} / \mathrm{ha})$} \\
\hline & \multicolumn{2}{|c|}{$\begin{array}{c}\text { Modelo } \\
\text { intermedio }\end{array}$} & \multicolumn{2}{|c|}{$\begin{array}{l}\text { Análisis } \\
\text { discreto }\end{array}$} & \multirow{2}{*}{\multicolumn{2}{|c|}{$\begin{array}{l}\text { Modelo básico } \\
\text { por localidad } \\
\mathrm{N}^{*} \quad \mathrm{P}_{2} \mathrm{O}_{5}^{*}\end{array}$}} \\
\hline & $N^{*}$ & $\mathrm{P}_{2} \mathrm{O}_{5}$ * & $N^{*}$ & $\mathrm{P}_{2} \mathrm{O}_{5}$ * & & \\
\hline $\begin{array}{l}\text { DR1, alto } \\
\text { P inicial, } \\
\text { baja MO. }\end{array}$ & 184 & 0 & 200 & 0 & 130 & 0 \\
\hline $\begin{array}{l}\text { DR2, bajo } \\
\text { P inicial, } \\
\text { alta Mo. }\end{array}$ & 72 & 51 & 50 & 80 & 50 & 86 \\
\hline
\end{tabular}
acuerdo a diferentes modelos.

* Significa dósis óptima.
Para fines comparativos se presentan en las columnas 4 y 5 las dosis óptimas económicas obtenidas mediante la aplicación del análisis discreto a los datos agrupados por dominio de recomendación con base en la variable $\mathrm{D}, \mathrm{y}$ en las dos últimas columnas aquellas obtenidas con el modelo básico estimado para cada una de las localidades.

Los resultados obtenidos del ajuste de la función cuadrática indican que para aquellos agricultores dentro del dominio de recomendación 1 (DR1) donde la MO es baja y el fósforo es aluo, los agricultores deberían a los precios vigentes duplicar la fertilización con $\mathrm{N}$ y no aplicar P. En cambio para el dominio de recomendación 2 (DR2) con alta $\mathrm{MO}$ y bajo $\mathrm{P}$ inicial, las dosis de $\mathrm{N}$ y $\mathrm{P}$ aplicadas por los agricultores, $80 \mathrm{kgN} / \mathrm{ha} \mathrm{y} 45 \mathrm{kgP}_{2} \mathrm{O}_{5} / \mathrm{ha}$, están cerca del óptimo por lo cual se las puede considerar adecuadas a los precios vigentes.

Dado que las dosis óptimas dependen de las relaciones de precios entre el precio de los nutrimentos y el del maíz $r_{n}$ y $r_{p}$, se realizó un análisis de sensibilidad considerando cambios en las relaciones de precios vigentes de 100, 50 y $30 \%$ tanto hacia arriba como hacia abajo. En el primer caso el nutrimento se hace mas caro respecto al maíz y vicerversa en el segundo caso. El cuadro 4 presenta los resultados del análisis.

Es posible observar que un aumento de $100 \%$ en las relaciones de precios $r_{n}$ y $r_{p}$ producen una reducción de alrededor del $60 \%$ en los beneficios netos por hectárea. Esta reducción es provocada por una disminución de las cantidades óptimas de nutrimentos en ambos dominios.

La reducción de las dosis varía con el nutrimento y el dominio, por ejemplo en el caso del $\mathrm{N}$ la dosis óptima puede reducirse a la mitad si los precios relativos de los fertilizantes aumentan al doble. En el caso improbable en que los precios relativos disminuyan la sensibilidad de nutrimentos y beneficios es mucho mayor. Por ejemplo si los precios relativos disminuyen en 33\%, los beneficios netos aumentan en $62 \%$ mientras que las cantidades óptimas de nutrimentos aumentan hasta $150 \%$ en el caso del P.

De la función de respuesta estimada y de las condiciones de primer grado para hallar las dosis óptimas se derivaron para cada uno de los dos dominios de recomendación, tres funciones que arrojan una perspectiva diferente al problema. Ellas son: el sendero de expansión, la funcion de costos inversa y la función de rentabilidad. El cálculo de estas funciones resulta 
engorrosa algebraicamente pero relativamente fácil de evaluar numéricamente usando una hoja electrónica de cálculo. El sendero de expansión da las proporciones óptimas de ambos nutrimentos para cada nivel de gasto total en fertilizante. La función de costo inversa expresa el rendimiento que se obtiene a medida que se incrementa el gasto en fertilizante cuando los nutrlmentos se combinan en proporciones óptimas, es decir a lo largo del sendero de expansión (Beattie y Taylor 1985). La función de beneficio neto es el equivalente continuo de la curva de beneficio neto obtenida en el análisis discreto, es decir que relaciona los beneficios netos que se obtendrian de cada nivel de gasto total en fertilizante, pero a diferencia del análisis discreto en esta función los nutrimentos están combinados en proporciones óptimas (Sain y Jauregui 1992). Finalmente, derivando la funci6n de beneficio neto (BN) respecto a TCV se obtiene la función de rentabilidad de la inversión en $\mathrm{N}$ y $\mathrm{P}$ medida a través de la tasa marginal de retorno (TMR) como función de la cantidad de gasto total en fertilizante.

Las siguientes ecuaciones estimadas muestran las las funciones del sendero de expansión, la función de costo inversa, y la función de beneficios netos para los dos dominios de recomendación.

\section{DR2. Bajo P inicial, alta MO}

Sendero de expansión

[10] $\mathrm{P}=-357+\mathrm{S}, 54 \mathrm{~N}$

Función de costo inversa

[11] $\mathrm{P}=-874+242(\mathrm{TCV})-2,82(\mathrm{TCV})^{2}$

Función de beneficio neto

[12] $\mathrm{BN}=-194+45,6(\mathrm{TCV})-0,559(\mathrm{TCV})^{2}$

Función de rentabilidad

[13] $\mathrm{TMR}=45,6-1,118(\mathrm{TCV})$

\section{DRI. Alto P Inicial, baja MO}

Sendero de expansión

$$
\mathrm{P}=-1089+5,54 \mathrm{~N}
$$

Función de costo inversa

$\mathrm{Y}=-14715+488,2(\mathrm{TCV})-2,82(\mathrm{TCV})^{2}$

Función de beneficio neto

$\mathrm{BN}=-2800+93,0(\mathrm{TCV})-0,559(\mathrm{TCV})^{2}$
Función de rentabilidad

$\mathrm{TMR}=93,0-1,118(\mathrm{TCV})$

Cuadro 4. Sensibilidad de las dosis óptimas de los nutrimentos y beneficios ante cambios en las relaciones de precios.

(a) Dominio recomendación 1. Alto P inicial, baja MO.

\begin{tabular}{lcccr}
\hline $\begin{array}{l}\text { Relaciones de precios } \\
r_{n}\end{array}$ & $r_{p}$ & $\begin{array}{l}\text { Dosis optimas } \\
N^{*}\end{array}$ & $\begin{array}{l}\text { Beneficios } \\
\text { (\$/ha) }\end{array}$ \\
\hline 8,19 & 12,08 & 147 & 0 & 419 \\
5,46 & 8,06 & 172 & 0 & 687 \\
4,82 & 7,11 & 177 & 0 & 795 \\
4,10 & 6,04 & 184 & 0 & 959 \\
3,56 & 5,25 & 189 & 0 & 1124 \\
3,28 & 4,83 & 191 & 0 & 1234 \\
2,73 & 4,03 & 196 & 0 & 1509 \\
\hline
\end{tabular}

(b) Dominio recomendación 2. Bajo P inicial, alta MO.

\begin{tabular}{lrrrr}
\hline $\begin{array}{l}\text { Relaciones de precios } \\
r_{n}\end{array}$ & $\begin{array}{l}\text { Dosis optimas } \\
r_{p}\end{array}$ & $N^{*}$ & $\begin{array}{l}\text { Beneficios } \\
\text { (S/ha) }\end{array}$ \\
\hline 8,19 & 12,08 & 34 & 0 & 264 \\
5,46 & 8,06 & 59 & 0 & 413 \\
4,82 & 7,11 & 65 & 0 & 476 \\
4,10 & 6,04 & 72 & 51 & 573 \\
3,56 & 5,25 & 77 & 67 & 672 \\
3,28 & 4,83 & 79 & 82 & 742 \\
2,73 & 4,03 & 85 & 111 & 926 \\
\hline
\end{tabular}

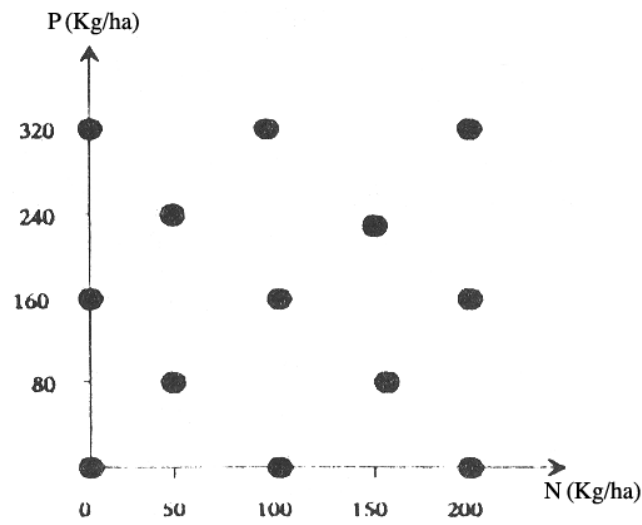

Figura 1. Arreglo factorial $5^{2}$ usado en el experimento

Es de notar que debido a la forma funcional cuadrática adoptada, las ecuaciones del sendero de expansión son dos líneas rectas que no pasan por el origen mientras que las funciones de costo inversa y de 


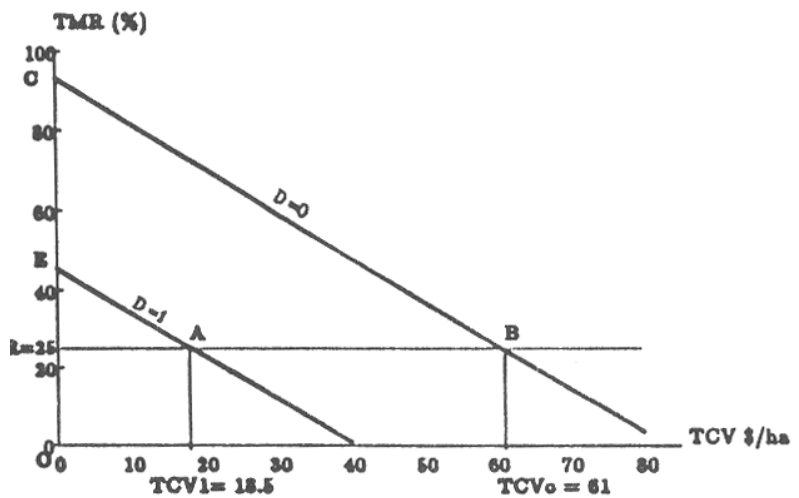

Figura 2. Funciones de rentabilidad de la inversión en fertilizantes en cada dominio de recomendación.

beneficios netos son iunciones cuadráticas en la variable TCV. En el caso de esta última función los interceptos negativos indican que la respuesta del cultivo es tal que la no inversión o no aplicación de fertilizantes resultaría en beneficios netos negativos.

La línea horizontal representa la tasa minima de retorno aceptable para los agricultores (R). Esta línea corta a las rentabilidades de la inversión en fertilizantes para los dos dominios en los puntos A y B. Por arriba de esos puntos la TMR es mayor que $\mathrm{R}$ y por 10 tanto convendrá seguir invirtiendo en fertilizante, mientras que por debajo la TMR es menor que $\mathrm{R}$ y la inversión no será redituable. Es decir que las cantidades invertidas $\mathrm{TCV}_{0} \mathrm{y}$ $\mathrm{TCV}_{1}$ son puntos de equilibrio de gastos óptimos en fertilizantes donde las proporciones de nutrimentos vienen dadas por las condiciones impuestas por el sendero de expansión.

Los valores del intercepto en estas ecuaciones indican los valores minimos de $\mathrm{R}$ necesarios para que ocurra inversión en fertilizantes. Por ejemplo si el costo del capital para los agricultores en el dominio de recomendación 2 estuviera por arriba del $42 \%$ entonces a los agricultores no les convendr la aplicar fertilizantes y si quisieran cultivar maíz deberian buscar fuentes alternativas mas baratas de nutrimentos.

\section{LITERATURA CITADA}

ACOSTA, M.; PINZON; B., RUIZ, J. 1991. Respuesta del maíz a la fertilización con nitrógeno y fósforo en tres suelos del suroeste de la Provincia de Chiriquí. Presentado en la XXXVII Reunión Anual del Programa Cooperativo Centroamericano para el Mejoramiento de Cultivos y Animales (PCCMCA). Panamá. 23 p.

BEATTIE, B.; TAYLOR, R. 1985. The economics of production. John Wiley \& Sons. New York. 320 p.

BYERLEE, D.; HARRINGTON, L. 1981. Deriving optimum fertilizer leve1s: the naive economist versus the practical farmer. International Maize and Wheat Improvement Center. Economics Program Training Note. Mexico. 14 p.

CENTRO INTERNACIONAL PARA EL MEJORAMIENTO DE MAIZ Y TRIGO. 1988. La formulación de recomendaciones a partir de datos agronómicos: Un manual metodológico de evaluación económica. Edición completamente revisada. México D.F., México. 79 p.

GUJARATI, D. 1992. Econometria. México, McGrawHill. 597 p. 2nd ed.

JAUREGUI, M.; SAIN, G. 1991. Continuous economic analysis of crop response to fertilizers in on-farm research. CIMMYT economics paper No 3. México D.F., México. 103 p.

SAIN, G.; JAUREGUI, M. 1992. Deriving fertilizer recomendations with a flexible functional form. Manuscrito enviado para su publicación en el Agronomy Journal. $16 \mathrm{p}$. 